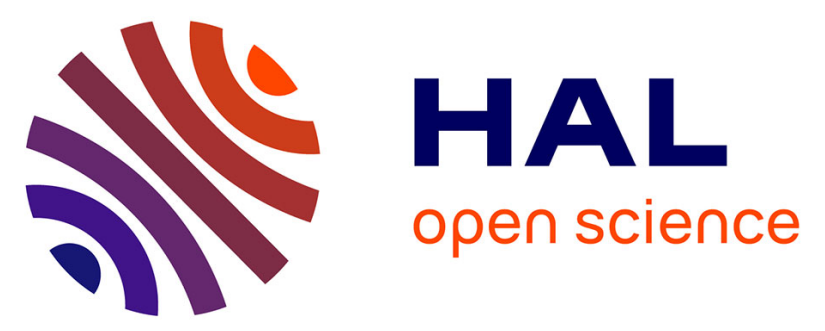

\title{
Crowdsourced Collaborative Decision Making in Crisis Management: Application to Desert Locust Survey and Control
}

Mohammed Benali, Abdessamed Réda Ghomari, Leila Zemmouchi-Ghomari

\section{- To cite this version:}

Mohammed Benali, Abdessamed Réda Ghomari, Leila Zemmouchi-Ghomari. Crowdsourced Collaborative Decision Making in Crisis Management: Application to Desert Locust Survey and Control. 6th IFIP International Conference on Computational Intelligence and Its Applications (CIIA), May 2018, Oran, Algeria. pp.533-545, 10.1007/978-3-319-89743-1_46 . hal-01913921

\author{
HAL Id: hal-01913921 \\ https://hal.inria.fr/hal-01913921
}

Submitted on 7 Nov 2018

HAL is a multi-disciplinary open access archive for the deposit and dissemination of scientific research documents, whether they are published or not. The documents may come from teaching and research institutions in France or abroad, or from public or private research centers.
L'archive ouverte pluridisciplinaire HAL, est destinée au dépôt et à la diffusion de documents scientifiques de niveau recherche, publiés ou non, émanant des établissements d'enseignement et de recherche français ou étrangers, des laboratoires publics ou privés.

\section{(c)(1)}

Distributed under a Creative Commons Attribution| 4.0 International License 


\title{
Crowdsourced Collaborative Decision Making in Crisis Management: Application to Desert Locust Survey and Control
}

\author{
Mohammed Benali, Abdessamed Réda Ghomari, and Zemmouchi-Ghomari \\ Leila
}

LMCS (Laboratoire des Méthodes de Conception des Systèmes), ESI (Ecole nationale Supérieure d'Informatique), Algiers, Algeria

$\{$ m_benali,a_ghomari,__zemmouchi\}@esi.dz

\begin{abstract}
Dealing with crisis situations involves significant collaborative decision-making to recover lives and preserve properties. In order to reach timely and appropriate decisions, crisis response organizations need to rapidly obtain an accurate situation awareness of the crisis context. A process by which they need to gather, access, and exchange near realtime information about the events circumstances throughout the entire crisis life cycle. Such activities imply a huge participation of collaborating organizations and goes even beyond their internal borders to reach the entire crisis-stricken community. Recent crisis and disaster situations have demonstrated the crucial role citizens can and must play in responding to such events. The growing development of advanced technologies has open the door for large public to be a key factor in making decisions and conducting guided response actions. We propose in this paper a comprehensive approach that integrates crisis crowdsourcing tasks and techniques to crisis decision-making activities. To demonstrate its relevance, we present a study of crisis scenario based on the context of Desert Locust Plague Survey and Control in the Algerian National Institute of Plant Protection (INPV).
\end{abstract}

Keywords: Crowdsourcing, Collaborative Decision-making, Crisis Management, Case Study, Desert Locust Survey and Control

\section{Introduction}

The dynamically changing environment of crisis events creates highly unpredictable and complex situations to be handled, with human lives and properties at stake. In such contexts, crisis response organizations look for an accurate situational awareness by retrieving and exchanging real-time contextual information and knowledge, share a collective response action plan for purpose of making efficient and well-informed decisions. Crisis management activities imply taking complex and collaborative decisions, involving a huge and diverse information and knowledge owned and distributed among a variety of contributing actors 
from different areas of interests and expertise [1]. Moreover, real crisis situations have shown that response activities exceed the capabilities of crisis response organizations staff members and resources and have compelled them to open their borders and bring up together the whole affected community including unskilled citizens. Moreover, studies have concluded that unexpected problems such as crisis and disaster cases involve giving the decision authority to those closest to the situation in either location or knowledge [2]. In fact, the growing development of collaborative technologies have shifted citizens role and participation during crisis events from being just victims watching and sharing posts about the event consequences using social media and social networks, to crisis responders involved in a more proactive contribution by performing specific tasks related to crisis decision-making. According to [3] crowdsourcing is a dynamic form of cooperative work intended for a broad range of citizens engaged in semi-autonomous tasks related to information management issues.

The main benefits of implementing crowdsourcing mechanism lie in its ability to promote civic involvement and connect it with organizations by facilitating active and collaborative problem solving and decision-making [4]. However, a remaining challenge lies on finding new ways of understanding, conceptualizing and defining specific design methodologies for aligning crisis crowd-tasking activities and techniques with respondent organizations decision-making processes. In addition, there is still more studies of real crisis scenarios to be conducted to analyze and examine the role that ordinary citizens may play in the different steps of decision-making activities in the whole crisis life cycle [5]. To address this challenge, we propose a comprehensive crowdsourced collaborative decisionmaking approach for the crisis management domain. To this end, we adopt the well-known decisional model IDC of Simon [6] composed of the three following phases: Intelligence (information gathering and sharing for problem identification and recognition), Design (generating alternative courses of action), Choice (evaluating, prioritizing, and selecting the best course of action). For each step of Simons decisional process, we specify the main crisis crowdsourcing tasks which the crowds are called to perform, and the appropriate technique for outsourcing those tasks. Regarding the collaborative nature of the crisis decision-making process, we use the BPMN4Social modeling notation [7], which is a social extension of Business Process Model and Notation (BPMN), that aims to define a specific notation for describing Social Business Process behaviors, to which we add some crowdsourcing related specifications.

In order to demonstrate the applicability of our approach in a practical scenario, we carry out a study of the Desert Locust Plague in the Algerian National Institute of Plant Protection (INPV). The mission of the INPV is to ensure sustained survey and control of desert locust situation across the entire Algerian territory with 2.4 million $\mathrm{km}^{2}$. To assess the current Desert Locust situation, plan further survey and control operations, and request external assistance, a variety of data and information are required. This information is provided by a set of collaborating organizations such as survey teams of INPV, the Food and Agricultural Organization (FAO), regional governmental organizations, and 
local humanitarian organizations. Given its limited staff members and resources compared to the vast area to be monitored, the INPV have to rely on additional information coming from less experienced people such as agricultural extension agents and scouts as well as from non-locust experts such as travelers, truck drivers, farmers, government agents, villagers and nomads. These features make the Desert Locust Plague a relevant case for the analysis of the challenges and opportunities that arise when integrating crowdsourcing processes to the participatory decision-making process of the collaborating stakeholders.

The reminder of this paper is structured as follows. Section 2 reviews the main related work addressing the crowdsourcing applicability to decision-making process. Section 3 presents the fundamental concepts of the proposed approach. Section 4 presents the evaluation of the approach by its application to the desert locust plague case. Finally, Section 5 concludes by summarizing the main contributions achieved in this research and makes recommendations for future work.

\section{Related Work}

Crowdsourcing have proven to be a promising field of research, giving the wideranging studies in which it has been addressed by scholars and practitioners over the last decade. In this work, we focus on the alignment of crowdsourcing process with organizational decision-making process in a crisis management context. Bonabeau [8] in his work provides a general framework to assess crowdsourcing application in business problem solving and decision-making. Considering that the decision-making is divided on two phases: the generation of potential alternatives and the evaluation of them, he discussed for each phase the main behavioral biases (social interference, availability, anchoring, and stimulation) that adversely influence the process, as well as the proper collective intelligence approaches (outreach, additive aggregation, and self-organization) to handle them. The crowdsourcing framework examine the key implementation issues such as control (loss of control, unpredictability, liability, etc.), diversity versus expertise, engagement (incentives to motivate people's participation), and policy (policies to control participants' behaviors). Another study we could mention is that carried out by [9]. In this study, a simulation experiment of the Citizens Emergency Response Portal System (CERPS) have been conducted, to examine the hypothesis that citizen participation in crisis response decision-making can improve the outcome of a crisis. The findings of this study have shown that citizen involvement provided additional first-hand situation awareness, such as the locations of fires or pictures of suspicious packages. The crowdsourced information helped crisis managers to make better response decisions, understand the population needs, and decide when and how to provide information to the public. Closer to our work, Chiu et al. [10] propose a crowdsourcing based decision-making framework. The framework specifies for each phase of the IDC model of Simon the main tasks to be crowdsourced. At the intelligence phase, the crowds are implicated in knowledge discovery activities, making predictions, and providing opinions. The crowds in the design phase, are called for idea and proposals gen- 
eration. At the choice phase, the main crowds' concern is the evaluation and assessment of the proposed ideas in the previous phase. Another relevant study in this topic is that conducted by [11]. They propose a crowdsourcing-based framework to build decision support systems based on the crowdsourced data. The proposed system provides on-the-fly decision support, and is based on two aspects: constructing the knowledge base in run-time using crowds' contributions and providing decision support using the collected knowledge. Another related study we mention is the proposal of [12] who propose an interactive crowdsourcing based decision support framework for post disaster situation awareness and decision support. The framework gathers situational information using SMS from the crowd present at the disaster site, and summarizes such responses to have situational awareness and appropriate decision-making.

Most of the prior works are oriented towards addressing the main issues and challenges facing organizations when incorporating crowdsourcing into their decision-making processes. In addition, most of the existing literature addresses the applicability of crowdsourcing to decision-making for general business problems and lacks more research that examines in particular the crisis management area. To the best of our knowledge current literature fails to establish a comprehensive approach to support the design of processes to integrate crowdsourcing into the different phases of crisis decision-making, which represents the main concern of this study.

\section{Crowdsourced Collaborative Decision-making Approach in Crisis Situations}

In this section, we delineate the way crowdsourcing processes can be incorporated into the decision-making process within a crisis management context. The crisis crowdsourcing approach is built upon cooperation between citizens and crisis response organizations (CROs), in each stage of the decision-making process at the various crisis management phases. For each phase of Simon's decisional model (IDC), citizens are engaged either by providing information about the crisis or as crowd workers performing specific tasks related to information processing and management issues. Thus, the proposed approach will allow response organizations to identify and express their information needs, be aware of the crisis evolving context, and strengthen their decision-making capabilities by providing relevant recommendations based on volunteer citizens' evaluations and feedbacks. As depicted in figure 1, the decisional process relies on a two-way flow of information, where crisis stakeholders may act both as consumers and as providers of information. From one hand, citizens provide near real-time information about the event to display an accurate situational awareness for the affected population and the involved response organizations. Respondent organizations, on the other hand, share their plans and resources to both citizens and the others crisis management organizations to share a clear activity awareness with the crisis management community and proceed to distributed planning and execution of response plans. 


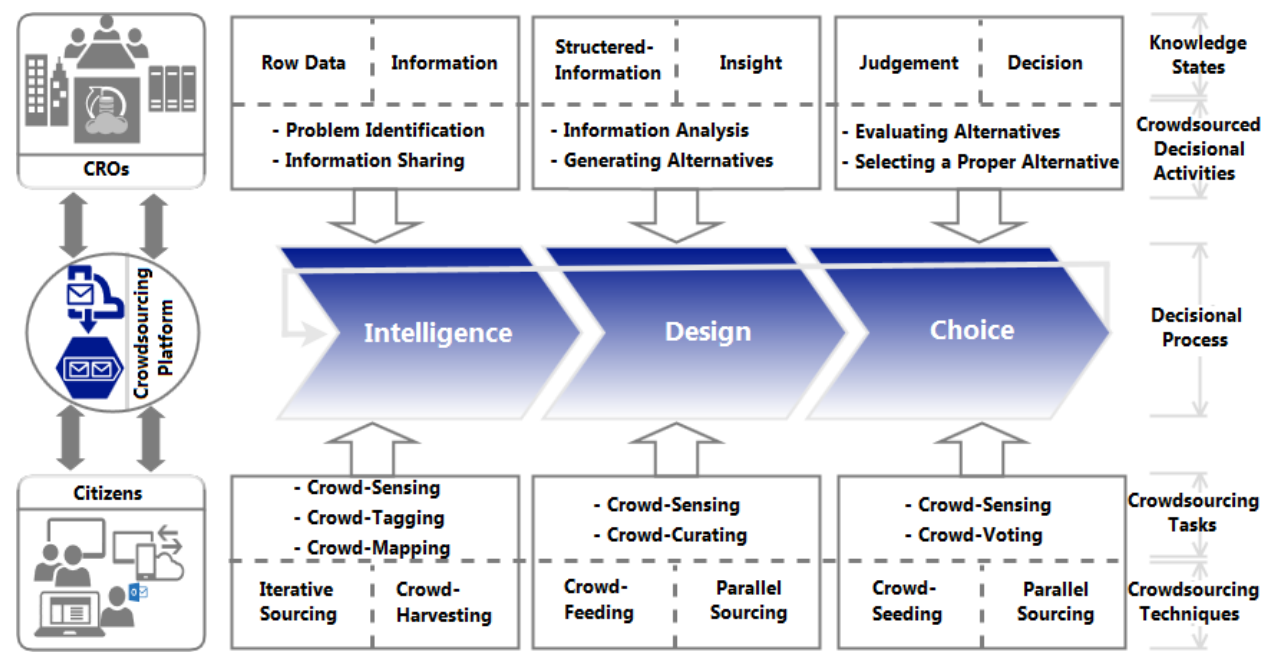

Fig. 1. Overview of the proposed Approach

\subsection{Phase I: Information Sharing and Problem Identification}

In this phase, the first concern of the respondent organizations is to rapidly obtain a clear on the ground picture of the affected zone. Maintaining an updated situation awareness of the crisis context requires gathering information from sensor systems in conjunction with crowdsourced data from citizens reporting observations and direct eyewitness accounts. In fact, local population tends to immediately gather and share geolocated data and information related to the crisis effects. They contribute to the information chain either passively by providing raw data using their phones, tablets, and social networking technologies or actively by reporting more established and controlled information using a dedicated crowdsourcing platform. Whereas this crowd-sensing activity represents a key pillar for response decisions, it may generate information overload problems, due to the great volume of the collected data and information. To overcome this challenge, crowd-tagging comes as an efficient means by which crowds can be involved in categorizing and classifying of the provided data based on their local knowledge and the perception of their own environment. Results of the tagging process are then presented and visualized to enhance the awareness of both response actors and the public. Response organizations, in turn, and based on the information harvested automatically from social networks or gathered via the platform, proceed to information categorization and classification using their internal expertise. In this regard, situational awareness ontologies are relevant tools used to avoid ambiguity and confusion of terms and share a common language with the different stakeholders, which have different cultures, protocols and systems. At the meanwhile, crowds iteratively improve these information mapping and tagging activities, by continuously reporting information according to the dynamically changing circumstances of the event. 


\subsection{Phase II: Generating possible Courses of Action}

At this stage of the decisional process, the primary objective of the crisis response organizations is to find actionable crisis information that translates into action plan. During this phase, the crowds are involved in a more active participation, which goes beyond the simple sharing of raw data to more elaborated and structured information. A process that makes crowd-tasking activities even more complicated for certain member of the crowd. After identifying the main tasks to be assigned to the crowd, response organizations and based on the complexity of the tasks and the available resources, proceed to the crowd-selection process. In addition, they share georeferenced data about their field teams positions as well as their associated resources, which enhance activity awareness of the different collaborating organizations, facilitate the distributed response planning, and guide the crowds participations. In this regard, mapping and tagging activities can facilitate and improve the crowds understanding of the different tasks to be performed by giving them a global picture of the response plan. Once crowds are selected, the platform accordingly, generates the necessary instances of tasks and automatically assign them to each selected member. The crowds and based on the available information, proceed to the crowd-curating activity. They are consequently involved in various information processing and management tasks such as information filtering and synthesizing to support respondent actors with practical insights and guidances for the development of potential alternative courses of actions.

\subsection{Phase III: Evaluating and Selecting a Proper Alternative}

In this phase, the objective behind engaging the crowds is to get evaluations, opinions, and suggestions to prioritize response actions and improve alternatives created in the previous phase. To this end, response organizations share the proposed response plan through the platform with the different response stakeholders. The shared plan provides relevant basis for the other involved organizations to adjust their priorities and ensure an optimal use of the available response resources. An overview of the response plan can be presented to citizens using the crowd-mapping technique to enhance their awareness and gain their confidence. Evaluations are then collected, analyzed, and reviewed by the internal experts of the response organizations. While ranking activity provides explicit information, some evaluation can include complex responses containing implicit information, which require more clarifications or additional work to be requested from the crowd. Thus, response organizations can ask for additional details and explanations about a specific contribution. Crowdsourced data or tasks performed by the crowd are processed and reviewed and then shared back to the crowd to get more adjustments and feedbacks. In this context, the collection process can be improved using the parallel sourcing technique, where participants evaluate the same alternative independently of other's evaluations, which reduces the probability for participants to be influenced and guided by previous works. 


\section{Case Study}

The desert locust plague is considered as one of the major threats to food security worldwide due to its devastating effects on crops and pastures, which potentially results in disturbing socio-economic consequences. Locust outbreaks have occurred on all continents except Antarctica and they can harm the livelihood and well-being of $10 \%$ of the worlds population [13]. In fact, the desert locust outbreak of 2003-2005 affected 8 million people, in over 20 countries mostly subSaharan Africa, with an estimated 80 to $100 \%$ of crops lost [14]. Besides the financial losses, the large amounts of chemical insecticides commonly used for control operations can result in serious environmental damage [14]. In Algeria, the last desert locust invasion, in 2004-2005, has required an urgent mobilization of financial resources estimated at USD 120 million to treat 4.5 million hectares which contains mainly hopper bands and swarms [15]. To cope with such a calamity, application of an effective prevention and control strategy is necessary to protect the agricultural production. In this regard, the National Institute of Plant Protection ${ }^{1}$ have been charged to take over this critical task, which aims to organize and conduct during the recession period the monitoring and control operations against the Desert Locust, develop and coordinate during the invasion period a response plan for control operations.

\subsection{Desert Locust Survey and Control Process at the INPV}

The National Institute of Plant Protection carries out a sustained survey and control of desert locust situation all over the Algerian territory with 2.4 million km2 and especially in the southern Saharan zone. Usually, monitoring and control operations are undertaken by qualified Locust Field Officers who send field reports to the national unit headquarter. Received reports are then corrected, stored, and visualized by the Locust Information Officer (LIO) using RAMSES (Reconnaissance and Monitoring System of the Environment of Schistocerca) a centralized system set up by the desert locust information service (DLIS) of FAO. In addition, the LIO receives information from the DLIS of the FAO which consists of a bulletin that contains a summary of the current situation at the locust-affected countries and a forecast of future developments. Despite the amount and the variety of collected data and information, experienced locust officers at the INPV assume that just $50 \%$ of information about the locust situation are detected by survey operations for a given area, due to the limited capacity of the INPV Staff regarding the vast area to be monitored and the accessibility of locust habitat. To strengthen its locust situational awareness, the INPV rely on additional information coming from less experienced people such as agricultural extension agents and scouts as well as from non-locust experts such as travelers, truck drivers, farmers, government agents, villagers and nomads. After verifying these information, the LIO incorporate meteorological

\footnotetext{
${ }^{1}$ http://www $\cdot$ inpv.edu.dz/
} 
and SPOT-vegetation data, assess the current situation and forecasts future developments. In order to have a global view of the locust situation, the LIO send a weekly (in the recession period) or monthly (in the invasion case) report to the DLIS of FAO which incorporate it to its locust bulletin. Citizens are also informed about the locust situation and the targeted areas of control campaigns. Based on the analysis performed by the LIO, the administrative board decides when and where to conduct survey operations, what are the priorities of control campaigns, evaluate the effectiveness of control operations, and determine where further control is required.

\subsection{Crowdsourcing the Decisional Process of the INPV}

\section{Information Sharing and Problem Identification}

A better understanding of the locust situation means better assessment of the current situation, which leads to accurate forecasts of future developments and make the basis of all locust response decisions. The LIO receives locust information from locust monitoring teams, which contain a detailed report of the surveyed area. Locust situation reports are also provided by the DLIS of FAO, which represents a global situational image of the locust affected countries. However, experienced locust field officers of the INPV suppose that these information cover only $50 \%$ of the overall locust situation. Especially in unsurveyed areas, non-locust experts such as villagers, nomads, travelers, truck drivers, and government agents play a crucial role in monitoring and reporting information about habitat conditions and locust presence. In addition to directly using the crowdsourcing platform, posts and comments on locust situation can be automatically collected incorporated from social networks to the platform using dedicated webbased sensors. Four key data are required: ecology, rainfall, locusts and control. As highlighted in figure 2, locals and based on their knowledge of their own environment, provide ecological data which comprise a description of the habitat nature (such as valleys, plains, pastures), density and the greenness of the vegetation, and soil moisture. In this regard, SPOT-vegetation data are provided by the Algerian Space Agency (ASA) to allow a guided citizens participation. They also provide meteorological data, which consists of Rainfall (to identify vegetation and breeding areas), temperature (to estimate the development rate of eggs and hoppers), wind and atmospheric pressure (to evaluate the possibility of adult development and their migrations direction). These data are compared with confirmed data from National Office of Meteorology (NOM). In addition, citizens can provide critical locust data such as locust maturity, density, appearance, phase, and behavior. To this end, mapping tools should be incorporated to the crowdsourcing platform to enable geo-locating and geo-tagging of the locust affected area. The LIO analyze the received reports and compare the analysis results with historical data to understand the dynamics of locust populations. Crowds can be asked to report particular pieces of information such as locust colors, pictures of the observed location, etc. Based on the analysis results, he evaluates the current locust situation and forecast future development. 


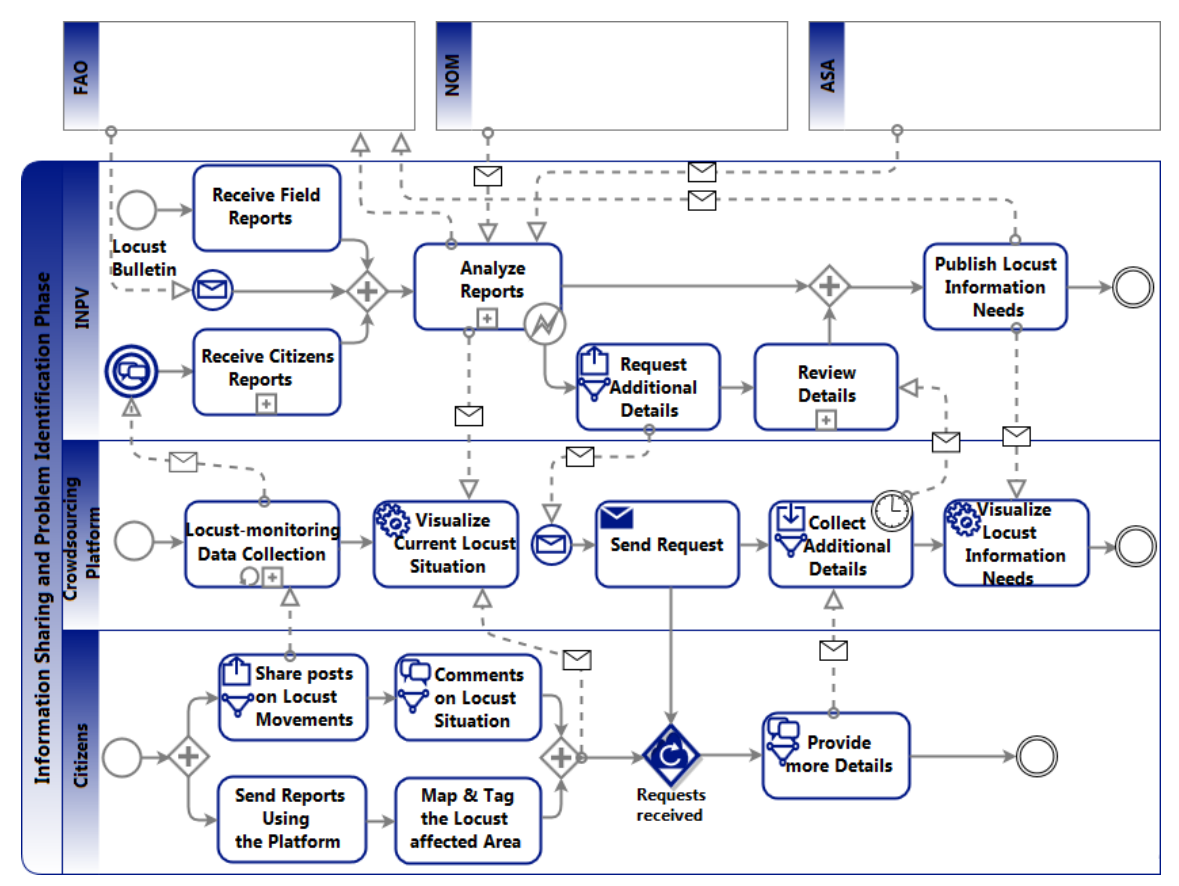

Fig. 2. BPMN4Social modeling of the Information Sharing and Problem Identification Phase

\section{Generating possible Courses of Action}

After identifying the main information to be collected, the INPV evaluate its internal staff capabilities and its own resources regarding the extent of the locust situation. Thereafter, the LIO identifies the main tasks to be crowdsourced based on situational reports analysis and collected contributions as depicted in figure 3 . in this regard, profile analysis techniques may be of great value in selecting crowd members and assigning tasks. Usually, the previous phase involves a large number of contributors providing near-real time eye-witnesses in form of raw data. The huge amount of crowdsourced data demand considerable work, time, and concerted efforts to extract actionable information used for locust forecasts and response planning. The main objective of the crowd is to reduce irrelevance and redundancy of information in received reports, analyze and synthesize posts and comments from social networks. Furthermore, the crowdsourcing platform should allow selected citizens to fill out pre-prepared forms such as selecting images of ecological conditions (plain, dunes, crops), rainfall (light, moderate, heavy), locust maturity (hopper instars, fledgling, immature, maturing, mature adult), locust behavior (isolated, scattered, copulating, laying, flying), and its size or density (very small, small, medium, large, very large). Contributions are then visualized to get feedbacks and improvements. A synthesized report is then sent to the INPV decision makers in order to prioritize the response operations, and elaborate a coordinated plan for exploitation and distribution of resources. 


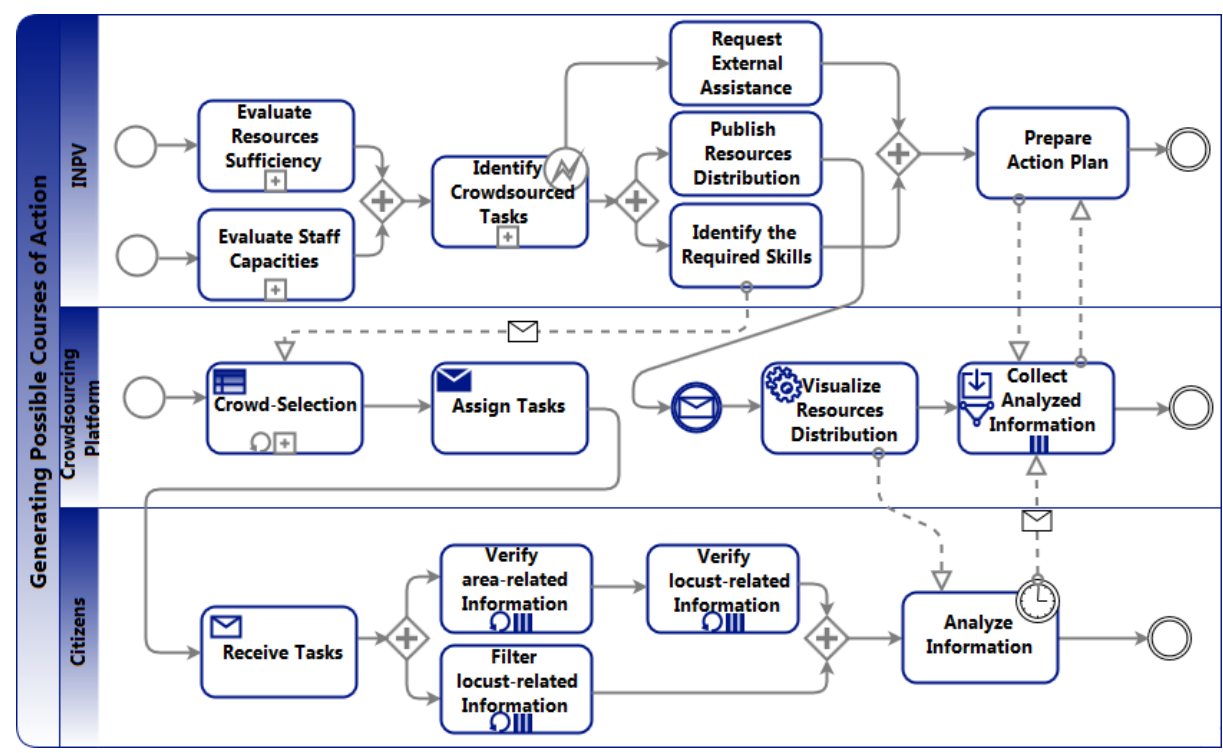

Fig. 3. BPMN4Social modeling of the Generating Possible Courses of Actions Phase

\section{Selecting the Appropriate Alternative}

The main preoccupation of the INPV in this phase is to generate a shared and distributed locust response action plan. To this end, the INPV locust experts prepare and share a draft action plan with selected crowds and the other concerned organizations such as the Agricultural Services Branch, General Direction of Forests, Civil Protection Services, and Local Communities. Using mapping features of the platform, the INPV in conjunction with the other involved organizations display geo-tag locations of risk areas, confirmed locust-related information, potential targets for intervention, means and control methods (mechanical control, baiting, dusting, and spraying), route itineraries, and the estimated time for arrival of the locust survey and control teams. For their part, citizens directly engage in crowd-voting activities, which involve commenting, ranking and evaluating of the proposed draft action plan. They can give feedbacks on particular points or alternatives and provide evaluation and action plan proposals. Evaluations are collected, analyzed, and reviewed by locust response teams, who may request additional details on citizens proposals such as confirmation about route itineraries, habitat conditions, and locust behavior. After reviewing citizens analysis, desert locust control commission prioritize response actions, prepare and implement the operational action plan. On the other hand, citizens continuously evaluate the effectiveness of the action plan progress and send feedbacks. According the changing circumstances of the locust situation and citizens insights and reactions to the response plan, locust response teams adjust the later as shown in figure 4 . 


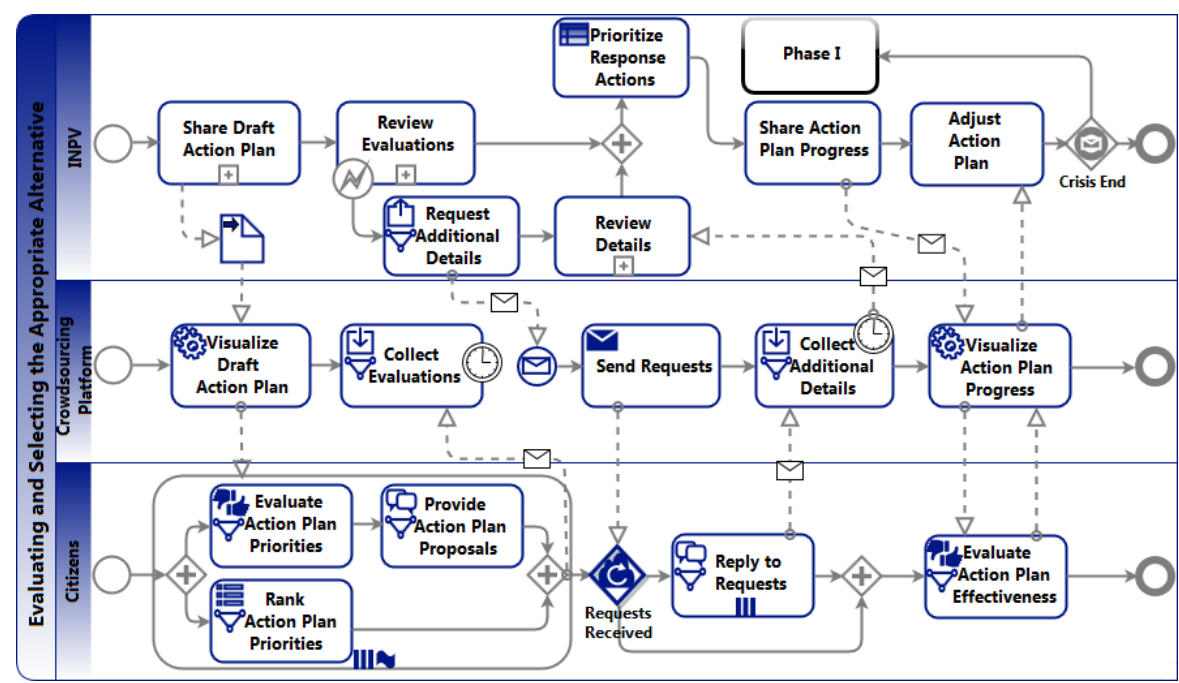

Fig. 4. BPMN4Social modeling of Evaluating and Selecting the Appropriate Alternative Phase

\section{Conclusion}

Handling crisis events implies collaborative, distributed and complex decisionmaking activities involving diverse sources of spatiotemporal information and knowledge, distributed among stakeholders from different organizations, agencies and even ordinary citizens. The increasing development of collaborative technologies has conducted crisis decision-making towards a more civic-driven process. Unfortunately, available literature lacks studies and design methodologies intended to align and leverage the emerging power of crowdsourcing to enhance organizational decision-making process especially in crisis situations. The contribution outlined in this paper is a comprehensive approach for crisis collaborative decision-making, which identifies the type of required information and data, and the main crowd-tasking activities and techniques for each phase of response organizations decisional process. Application of the approach in the context of a real crisis situation at the INPV has demonstrated the potential of the approach as an underlying basis to crowdsource information management and processing needs throughout the crisis life cycle. Furthermore, application to this case study also showed that the central aspect of the crowdsourcing platform role is to ensure detection of early warning signals of the locust plague by crowd-harvesting data from social networks or directly incorporating citizens contributions and provide near-real time spatial visualization of the locust situation. In addition, it allows the coordinated planning and evaluation of response operations by either the crowd providing feedbacks and proposals or the other involved organizations by visualizing their resources and sharing their field expertise.

Our next steps will be to implement and test the described platform on a simulated locust recession and invasion event to evaluate its feasibility. Furthermore, 
we intend to increase the approach flexibility with more modeling specifications in order to ensure its applicability to other crisis situations.

\section{References}

1. Poblet, M., García-Cuesta, E., Casanovas, P.: Crowdsourcing roles, methods and tools for data-intensive disaster management. Information Systems Frontiers (2017) 1-17

2. Turoff, M., White, C., Plotnick, L., Hiltz, S.R.: Dynamic emergency response management for large scale decision making in extreme events. In: Proceedings of the 5th International ISCRAM Conference, ISCRAM Washington, DC, USA (2008) 462-470

3. Liu, S.B.: Crisis crowdsourcing framework: Designing strategic configurations of crowdsourcing for the emergency management domain. Computer Supported Cooperative Work (CSCW) 23(4-6) (2014) 389-443

4. Brabham, D.C., Ribisl, K.M., Kirchner, T.R., Bernhardt, J.M.: Crowdsourcing applications for public health. American journal of preventive medicine 46(2) (2014) 179-187

5. Benali, M., Ghomari, A.R.: Towards a crowdsourcing-based approach to enhance decision making in collaborative crisis management. In: Proceedings of the 14th International ISCRAM Conference, ISCRAM Albi, France (2017) 554-563

6. Simon, H.A.: Administrative behavior. Volume 3. Cambridge University Press, Cambridge (1976)

7. Yahya, F., Boukadi, K., Maamar, Z., Abdallah, H.B.: Enhancing business processes with web 2.0 features. In: e-Business and Telecommunications (ICETE), 2015 12th International Joint Conference on. Volume 2., IEEE (2015) 183-190

8. Bonabeau, E.: Decisions 2.0: The power of collective intelligence. MIT Sloan management review $\mathbf{5 0}(2)$ (2009) 45

9. Laskey, K.B.: Crowdsourced decision support for emergency responders. Technical report, George Mason univ FairFax VA Center for Excellence In Command Control Communications Computers-Intelligence (2013)

10. Chiu, C.M., Liang, T.P., Turban, E.: What can crowdsourcing do for decision support? Decision Support Systems 65 (2014) 40-49

11. Hosio, S., Goncalves, J., Anagnostopoulos, T., Kostakos, V.: Leveraging wisdom of the crowd for decision support. In: Proceedings of the 30th International BCS Human Computer Interaction Conference: Fusion!, BCS Learning \& Development Ltd. (2016) 38

12. Basu, M., Bandyopadhyay, S., Ghosh, S.: Post disaster situation awareness and decision support through interactive crowdsourcing. Procedia Engineering 159 (2016) $167-173$

13. Latchininsky, V.: Locusts. In: Encyclopedia of Animal Behavior, M. D. BreedJ. Moore, Eds. Volume 2. (2010) 288-297

14. Brader, L., Djibo, H., Faye, F., Ghaout, S., Lazar, M., Luzietoso, P., Babah, M.O.: Towards a more effective response to desert locusts and their impacts on food security, livelihoods and poverty. Multilateral evaluation of the 2003-05 Desert locust campaign. Food and Agriculture Organisation, Rome (2006)

15. LAZAR, M.: La dynamique des populations du criquet pèlerin (Schistocerca gregaria, Forsk. 1775) dans ses aires grégarigènes du sud algérien. Apport des données historiques et satellitaires pour améliorer la prévision des pullulations. $\mathrm{PhD}$ thesis, ENSA (2015) 